\title{
Original
}

\section{Clinicopathologic Significance of Galectin-3 Expression Patterns in Gastric Cancer Patients}

\author{
Shun SATO ${ }^{1,2)}$, Nobuyuki OHIKE ${ }^{3)}$, Tetsuji EnOsaWA ${ }^{4)}$, \\ Masashi Sato ${ }^{1)}$, Hiromi ImatakA ${ }^{1)}$, Yukihiro WadA ${ }^{1)}$, \\ Tomokazu Hisayuki ${ }^{1)}$, Hiromi Date ${ }^{1)}$, Tomoaki Mori ${ }^{1)}$, \\ Syuei Arima ${ }^{1)}$, Kouji SaIto ${ }^{1)}$, Shigeharu Hamatani ${ }^{1,3)}$, \\ Toshiaki Kunimura ${ }^{1)}$, Masaaki Matsukawa ${ }^{1,2)}$ and Toshio Morohoshi ${ }^{1)}$
}

\begin{abstract}
We evaluated the galectin-3 expression patterns and their clinicopathologic significance in patients with gastric cancer. The study included 89 patients with gastric cancer that had been surgically resected. Based on Lauren' s classification, 36 patients had intestinal-type cancer and 53 patients had diffuse-type cancer. Lymph node metastasis was observed in 47 patients. The expression of galectin-3 was determined immunohistochemically. Galectin-3 expression was observed in all 89 patients. Comparison of the groups of patients showing a high expression level of galectin-3 (high-expression group) and a low level of galectin-3 expression (low-expression group) showed that the low-expression group had significantly poorly differentiated tumors $(\mathrm{P}=$ $0.0223)$, more advanced histologic $\mathrm{T}$ factor $(\mathrm{P}=0.0236)$, and higher rates of lymph node metastasis $(\mathrm{P}=0.0003)$. Moreover, when the staining patterns in the high-expression group were classified as diffuse or scattered, significantly poorly differentiated tumors $(\mathrm{P}<0.0001)$, more advanced histologic $\mathrm{T}$ factor $(\mathrm{P}=0.0165)$, and higher rates of lymph node metastasis were observed in the subgroup with the scattered pattern of staining. Moreover, for intestinal-type cancer in the high-expression group, the rate of lymph node metastasis was significantly higher $(\mathrm{P}=0.001)$ in the scattered staining pattern subgroup. Our results suggest that tumor growth and metastasis rate are low if galectin-3 is expressed widely and diffusely in the tumor, and that the function of galectin-3 is to inhibit cancer growth. In contrast, prognosis is poor when galectin-3 is expressed in either a small area or over a large area but in a scattered pattern.
\end{abstract}

Key words : gastric cancer, galectin-3, immunohistochemistry, lymph node metastasis

1) First Department of Pathology, Showa University School of Medicine, 1-5-8 Hatanodai, Shinagawa-ku, Tokyo 1428555, Japan.

2) Department of Internal Medicine, Showa University Toyosu Hospital

3) Department of Pathology, Showa University Northern Yokohama Hospital

4) Department of Pathology, Showa University Fujigaoka Hospital 


\section{Introduction}

Gastric cancer is more prevalent in East Asia than in Europe or the United States. The number of deaths from gastric cancer in Japan stands at 50,000 annually, which ranks it second among all types of lethal cancer for both men and women. The prognosis for patients with gastric cancer largely depends on the cancer stage at diagnosis, histologic type, and the presence or absence of lymph node metastasis. Recently, while the number of gastric cancer cases in which the patients' lives can be saved has been increasing owing to improvements in diagnostics, there are still many cases of late-stage gastric cancer and recurrent cancer. Palliative surgery and multimodality therapy using S-1 or combination chemotherapy with S-1 are performed for incurable and unresectable cases in Japan, even though the effectiveness of these therapies is insufficient ${ }^{1,2)}$. Therefore, it is necessary to elucidate further the pathologic conditions of gastric cancer and to develop new treatment strategies.

Galectin-3 is implicated in tumor malignancy. Galectin is one of the intrinsic $\beta$-galactoside-binding animal proteins present in many tissue and cell types; 14 galectin families have been identified to date ${ }^{3,4}$. Of these, galectin-3 is considered to exert biologic functions as a tissue differentiation factor, cell-matrix adhesion factor, and pro-apoptotic factor ${ }^{5-7)}$. The relationship between galectin-3 expression and prognosis for various types of cancer has been studied using clinical specimens ${ }^{8-20)}$, and some of these studies have indicated that a low level of galectin-3 expression correlates with poor prognosis and lymph node metastasis in patients with pancreatic cancer, prostate cancer, breast cancer, and ovarian cancer ${ }^{11-14)}$. Thus, it appears that the biologic functions of galectin-3 are important for cancer progression. Although there have been similar reports concerning gastric cancer, contradictory results have also been reported, and a thorough understanding of galectin-3 in gastric cancer has not yet been reached ${ }^{15-18)}$. The aims of the present study were to examine the galectin-3 expression patterns in patients with resected gastric cancer in our hospital, and to determine the clinicopathologic significance of galectin-3 expression. In particular, we classified the expression patterns of galectin-3 and examined the relationships between the galectin-3 expression patterns and clinicopathologic prognostic factors.

\section{Materials and Methods}

\section{Tissue Specimens}

The subjects were 89 patients with gastric cancer that was surgically resected at Showa University Hospital in the period from May 2004 to October 2005. Patients with multiple primary cancers and those with tumors that could not be histologically staged were excluded from the study. The subjects were 53 men and 36 women with a mean age of $66.2 \pm 10.5$ years (range, 39-85 years). The depth of each tumor and the degree of histodifferentiation were determined by preparing a specimen with the maximum cut surface, whereby the section passes through the deepest part of the tumor, followed by staining with hematoxylin 
and eosin (HE). The tumors were classified with respect to the degree of histodifferentiation, i.e., the intestinal type and diffuse type of Lauren's classification, and the pathologic stage was determined in accordance with the TNM classification (UICC, 5th edition) ${ }^{21,22)}$.

\section{Immunohistochemical Analysis of Galectin-3}

The antibody used for immunohistochemistry was an anti-galectin-3 antibody (Santa Cruz Biotechnology) diluted 1:100 in mild CC1 (EDTA). The specimens were incubated with the anti-galectin-3 antibody for $1 \mathrm{~h}$. Galectin-3 was stained using the Ventana Automated system.

\section{Evaluation of Galectin-3 Staining}

The galectin-3 expression patterns were determined under an optical microscope. The frequency of galectin-3-positive cancer cells was evaluated using the method of Okada et $a l^{18)}$. Briefly, the number of galectin-3-positive cells was counted using a grid in five arbitrary fields under $100 \times$ magnification. Moreover, on the basis of the finding that the mean rate of galectin-3-positive cells in 89 patients was $49.7 \%$, patients with $\geq 50 \%$ galectin3 -positive tumor cells were classified into the 'high-expression' group and those with $<50 \%$ galectin-3-positive tumor cells were classified into the 'low-expression' group. Subsequently, the galectin-3 staining patterns of the high-expression group were analyzed. When multiple expression patterns were observed in one lesion, the expression pattern that predominated in $50 \%$ or more of the entire tumor was adopted.

\section{Statistical Analysis}

A multigroup analysis of the galectin-3 expression patterns and various clinicopathologic factors was performed using the $\chi^{2}$ test, and a difference with $\mathrm{P}<0.05$ was considered significant.

\section{Results}

\section{Clinicopathologic Characteristics of the Patients}

When the degree of tumor differentiation was determined histopathologically, it was found that 37 patients $(41.6 \%)$ had the intestinal-type and 52 patients $(58.4 \%)$ had the diffuse-type of cancer. For classification based on tumor depth, 33 patients were at pT1, 32 at pT2, 21 at pT3, and 3 at pT4. Lymph node metastasis was observed in 47 patients $(52.8 \%)$. Regarding histologic stage, 41 patients were in stage I, 21 patients in stage II, 13 patients in stage III, and 14 patients in stage IV of the disease.

\section{Expression of Galectin-3 and Prognostic Factors}

Galectin-3 expression was detected in all 89 patients, with 55 patients being classified into the high-expression group and 34 patients being assigned to the low-expression group. Table 1 
Table 1. Correlations between galectin-3 expression and the clinicopathologic findings

\begin{tabular}{lccc}
\hline & \multicolumn{2}{c}{ Galectin-3 } & \multirow{2}{*}{$P$} \\
\cline { 2 - 3 } & High & low & \\
\hline Lauren type & & & 0.0223 \\
$\quad$ Intestinal & 28 & 9 & \\
Diffuse & 27 & 25 & \\
Tumor status & & & 0.0236 \\
pT1 & 26 & 7 & \\
pT2 & 20 & 12 & \\
pT3 & 8 & 13 & \\
pT4 & 1 & 2 & 0.0003 \\
Nodal status & & & \\
pN- & 34 & 8 & 0.0002 \\
pN+ & 21 & 26 & \\
Pathologic stage & & & \\
I & 34 & 7 & \\
II & 12 & 9 & \\
III & 6 & 7 & \\
IV & 3 & & \\
\hline
\end{tabular}

shows the relationships between the two groups and the various clinicopathologic factors. A significantly lower degree of histodifferentiation $(\mathrm{P}=0.0223)$, more advanced histologic $\mathrm{T}$ factor $(P=0.0236)$, higher rate of lymph node metastasis $(P=0.0003)$, and more advanced histologic stage $(\mathrm{P}=0.0002)$ were observed in the low-expression group, as compared with the high-expression group.

\section{Galectin-3 Expression Patterns and Prognostic Factors in The High-Expression Group}

The galectin-3 staining patterns in the cytoplasm of tumor cells were classified as diffuse or scattered. The diffuse staining pattern was characterized by strong staining at the apical edge of the cytoplasm and staining of the entire cytoplasm (Fig. 1a, b). In contrast, a pattern of patchy positive staining scattered around the cytoplasm and a mixture of positive and negative staining in either a single tumor or a single tumor gland led to the designation of scattered staining (Fig. 1c).

Of the 55 patients in the high-expression group, 21 showed the diffuse staining pattern and 34 showed the scattered staining pattern. A significantly lower degree of histodifferentiation $(\mathrm{P}<0.0001)$, higher histologic $\mathrm{T}$ factor $(\mathrm{P}=0.0165)$, higher rate of lymph node metastasis $(\mathrm{P}=0.0033)$, and more advanced histological stage $(\mathrm{P}=0.0299)$ were observed in patients with the scattered staining pattern, as compared with patients with the diffuse staining pattern (Table 2). In addition, among the 26 patients at pT1 in the high-expression 


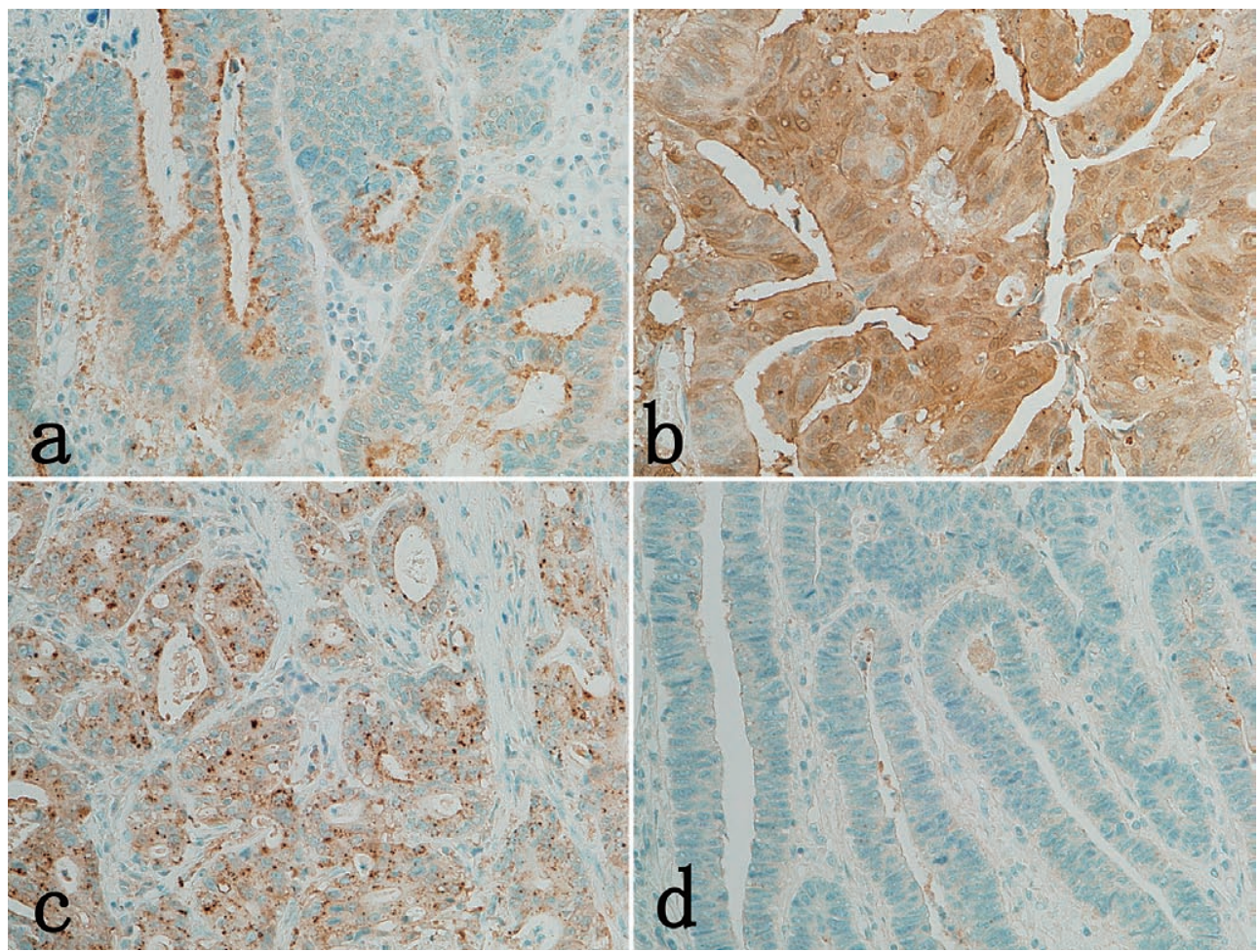

Fig. 1. Immunohistochemistry of galectin-3 in gastric cancer

a, b) In the diffuse pattern of staining for galectin-3, the edges of the cells are strongly stained (a, upper left), while the cytoplasm is diffusely stained ( $b$, upper right)

c) In the scattered pattern of staining for galectin-3, patchy positive staining is observed (lower left)

d) Negative staining (lower right)

group, lymph node metastasis was not observed in the 16 patients who showed the diffuse staining pattern. However, lymph node metastasis was observed in $20 \%(2 / 10)$ of the patients who showed the scattered staining pattern (Table 3).

Galectin-3 Expression Patterns in the Cytoplasm and Prognostic Factors for Patients with Intestinal-Type Cancer Showing High-Level Expression of Galectin-3

In the high-expression group, 27 patients had intestinal-type cancer. Among these patients, 20 showed the diffuse staining pattern and 7 showed the scattered staining pattern. Lymph node metastasis was observed at a significantly higher rate in those patients with the scattered staining pattern $(\mathrm{P}=0.001)$ (Table 4$)$.

\section{Expression of Galectin-3 in Normal Stomach Mucosal Tissue}

The level of galectin-3 expression was low in the normal foveolar epithelium, including the cell proliferative zone, as well as in the proper gastric glands. 
Table 2. Correlations between the galectin-3 expression patterns and clinicopathologic findings in the high galectin-3 expression group

\begin{tabular}{lccc}
\hline & High galectin-3 expression group & \multirow{2}{*}{$P$} \\
\cline { 2 - 3 } & Diffuse & Scattered & \\
\hline Lauren type & & 7 & \\
$\quad$ Intestinal & 20 & 27 & \\
Diffuse & 1 & & 0.0001 \\
Tumor status & & 10 & \\
pT1 & 16 & 16 & \\
pT2 & 4 & 7 & \\
pT3 & 1 & 1 & 0.0033 \\
pT4 & 0 & & \\
Nodal status & & 16 & \\
pN- & 18 & 18 & 0.0299 \\
pN+ & 3 & & \\
Pathologic stage & & 16 & \\
I & 18 & 5 & \\
II & 2 & 3 & \\
III & 1 & & \\
IV & 0 & & \\
\hline
\end{tabular}

Table 3. Correlations between the galectin-3 expression patterns and nodal status of pT1 gastric cancer in the high galectin-3 expression group

\begin{tabular}{cccc}
\hline & \multicolumn{2}{c}{ High gal-3 expression group } & \multirow{2}{*}{$P$} \\
\cline { 2 - 3 } & Diffuse & Scattered & \\
\hline Nodal status & & & 0.0726 \\
$\mathrm{pN}-$ & 16 & 8 & \\
$\mathrm{pN}+$ & 0 & 2 & \\
\hline
\end{tabular}

\section{Discussion}

Galectin is one of the intrinsic $\beta$-galactoside-binding animal proteins found in many tissues and cells; 14 galectin families have been identified to date ${ }^{3,4)}$. Among these, galectin-3 is considered to have biologic functions as a tissue differentiation factor, cellmatrix adhesion factor, and pro-apoptotic factor ${ }^{5-7)}$. Some studies have shown that a high expression level of galectin-3 correlates negatively with a poor prognosis and lymph node metastasis in pancreatic cancer, prostate cancer, breast cancer, and ovarian cancer ${ }^{11-14)}$. Although there are similar reports concerning gastric cancer ${ }^{18)}$, Miyazaki et al reported that galectin-3 was highly expressed in the nuclear rather than the cytoplasmic compartment 
Table 4. Correlations between the galectin-3 expression patterns and clinicopathologic findings of intestinal-type gastric cancer in the high galectin-3 expression group

\begin{tabular}{lccc}
\hline & \multicolumn{2}{c}{ High galectin-3 expression group } & \multirow{2}{*}{$P$} \\
\cline { 2 - 3 } & Diffuse & Scattered & \\
\hline Tumor status & & & 0.0654 \\
pT1 & 15 & 1 & \\
pT2 & 4 & 5 & \\
pT3 & 1 & 1 & 0.001 \\
pT4 & 0 & 0 & \\
Nodal status & & & \\
pN- & 17 & 1 & 0.0948 \\
pN+ & 3 & 6 & \\
Pathologic stage & & & \\
I & 17 & 2 & \\
II & 2 & 2 & \\
III & 1 & 1 & \\
IV & 0 & & \\
\hline
\end{tabular}

in poorly differentiated cancer, at that the high expression level of galectin-3 correlated positively with advanced stage disease ${ }^{16}$. These results suggest that the cellular localization of galectin-3 plays an important role in malignant transformation. Baldus et al ${ }^{17)}$ reported three variations of the cytoplasmic galectin-3 expression pattern in gastric cancer, namely, the cytoplasmic, luminal membrane, and Golgi-like expression patterns. However, they did not discuss the potential associations between the cytoplasmic galectin-3 expression patterns and tumor progression. There has been no study on the relationship between galectin-3 expression and gastric cancer prognosis.

The present study indicates that a low level of cytoplasmic expression of galectin-3 is associated with poor differentiation, risk of lymph node metastasis, and poor prognosis of gastric cancer, as is the case with many other types of cancer. In addition, for tumors with a high-level expression of galectin-3, a lower degree of histodifferentiation, more advanced tumor, and significantly higher rate of lymph node metastasis were observed in the scattered staining pattern group than in the diffuse staining pattern group. Similar findings with respect to staining patterns were obtained for intestinal-type cancer. For cases of gastric cancer in which galectin-3 was expressed at a high level, the growth and metastasis rate were low for those tumors in which galectin-3 was expressed diffusely over a large area. In contrast, tumor growth and the metastasis rate were high for those tumors in which galectin-3 was expressed in a confined area or in which galectin-3 was expressed in a scattered manner over a large area. These results appear to reflect the biologic functions of galectin-3, e.g., in apoptosis and cell adhesion. It seems that the cytoplasmic localization of 
galectin-3 expression causes the destabilization of cell adhesion.

It is not clear whether the galectin-3 expression pattern changes with tumor progression or whether the tumor has a diffuse pattern or scattered pattern of staining when the cancer first appears. However, it is assumed that some tumors present the scattered pattern at an early stage of disease, since tumors with the scattered pattern were observed in patients with pT1 tumors at a frequency of $38.5 \%(10 / 26)$.

Many early-stage cancer patients show evidence of lymph node metastasis. In the present study, although lymph node metastasis was not observed in the patients at $\mathrm{T} 1$ (which is defined as early-stage gastric cancer) who showed a diffuse staining pattern, it was observed in $20 \%$ of the $\mathrm{T} 1$ patients who had a scattered staining pattern. Therefore, early determination of the galectin-3 expression patterns in patients with gastric cancer might facilitate decisions as to therapeutic approaches, predictions of prognosis, and applications of chemotherapy.

\section{Conclusions}

The expression of galectin-3 correlates negatively with a poor prognosis of gastric cancer. In addition, even in patients with a high-level expression of galectin-3, the frequency of lymph node metastasis was significantly higher in patients with tumors that showed a scattered staining pattern for galectin-3. In summary, galectin-3 may be a useful maker of tumor progression and potential for lymph node metastasis, especially in patients with intestinal-type gastric cancer.

\section{Acknowledgments}

We are deeply grateful to Ms. Tomoko Nagai (First Department of Pathology, Showa University School of Medicine) for assistance with the preparation of specimens and immunostaining.

\section{References}

1) Sakata $Y$, Ohtsu A, Horikoshi N, Sugimachi K, Mitachi $Y$ and Taguchi $T$ : Late phase II study of novel oral fluoropyrimidine anticancer drug S-1 ( $1 \mathrm{M}$ tegafur-0.4 M gimestat-1 M otastat potassium) in advanced gastric cancer patients. Eur J Cancer $34: 1715-1720$ (1998)

2) Koizumi W, Tanabe S, Saigenji K, Ohtsu A, Boku N, Nagashima N, Shirao K, Matsumura $Y$ and Gotoh M: Phase I/II study of multimodel therapy with S-1 in patients with advanced gastric cancer. Br $J$ Cancer 89 : 2207-2212 (2003)

3) Barondes SH, Cooper DNW, Gitt MA and Leffler H: Galectins : structure and function of a large family of animal lectins. J Biol Chem 269 : 20807-20810 (1994)

4) Kasai K and Hirabayashi J : Galectins : a family of animal lectins. J Biochem 119 : 1-8 (1996)

5) Kuwabara I and Liu FT: Galectin-3 promotes adhesion of human neutrophils to laminin. J Immunol 156: 3939-3944 (1996)

6) Perillo NL, Marcus ME and Baum LG: Galectins : versatile modulators of cell adhesion, cell proliferation, and cell death. J Mol Med $76:$ 402-412 (1998)

7) Yang RY and Liu FT: Galectins in cell growth and apoptosis. Cell Mol Life Sci 60 : 267-276 (2003)

8) Herrmann ME, LiVolsi VA, Pasha TL, Roberts SA, Wojcik EM and Baloch ZW: Immunohistochemical expression of galectin-3 in benign and malignant thyroid lesions. Arch Pathol Lab Med 126:710-713 (2002) 
9) Takenaka Y, Inohara H, Yoshii T, Oshima K, Nakahara S, Akahani S, Honjo Y, Yamamoto Y, Raz A and Kubo T: Malignant transformation of thyroid follicular cells by galectin-3. Cancer Lett 195: 111-119 (2003)

10) Honjo Y, Inohara H, Akahani S, Yoshii T, Takenaka Y, Yoshida J, Hattori K, Tomiyama Y, Raz A and Kubo $\mathrm{T}$ : Expression of cytoplasmic galectin-3 as a prognostic marker in tongue carcinoma. Clin Cancer Res 6:46354640 (2000)

11) Shimamura T, Sakamoto M, Ino Y, Shimada K, Kosuge T, Sato Y, Tanaka K, Sekihara H and Hirohashi S: Clinicopathological significance of galectin-3 expression in ductal adenocarcinoma of the pancreas. Clin Cancer Res 8 : 2570-2575 (2002)

12) Pacis RA, Pilat MJ, Pienta KJ, Wojno K, Raz A, Hogan V and Cooper CR : Decreased galectin-3 expression in prostate cancer. Prostate $44: 118-123$ (2000)

13) Castronovo V, Van Den Brule FA, Jackers P, Claussee N, Liu FT, Gillet C and Sobel ME: Decreased expression of galectin-3 is associated with progression of human breast cancer. J Pathol 179: 43-48 (1996)

14) van den Brule FA, Buicu C, Berchuck A, Bast RC, Deprez M, Liu FT, Cooper DN, Pieters C, Sobel ME and Castronovo V: Expression of the 67-kD laminin receptor, galectin-1, and galectin-3 in advanced human uterine adenocarcinoma. Hum Pathol 27 : 1185-1191 (1996)

15) Lotan R, Ito H, Yasui W, Yokozaki H, Lotan D and Tahara E: Expression of a 31-kD lactoside-binding lectin in normal human gastric mucosa and in primary and metastatic gastric carcinomas. Int $J$ Cancer 56:474-480 (1994)

16) Miyazaki J, Hokari R, Kato S, Tsuzuki Y, Kawaguchi A, Nagao S, Itoh K and Miura S : Increased expression of galectin-3 in primary gastric cancer and the metastatic lymph nodes. Oncol Rep 9: 1307-1312 (2002)

17) Baldus SE, Zirbes TK, Weingarten M, Fromm A, Glossmann J, Hanisch FG, Monig SP, Schroder W, Flucke U, Thiele J, Holscher AH and Dienen HP: Increased galectin-3 expression in gastric cancer: correlations with histopathological subtypes, galactosylated antigens and tumor cell proliferation. Tumor Biol 21 : 258-266 (2000)

18) Okada K, Shimura T, Suehiro T, Mochiki E and Kuwano H: Reduced galectin-3 expression is an indicator of unfavorable prognosis in gastric cancer. Anticancer Res $26: 1369-1376$ (2006)

19) Lotz MM, Andrews CW Jr, Korzelius CA, Lee EC, Steele GD Jr, Clarke A and Mercurio AM: Decreased expression of Mac-2 (carbohydrate binding protein 35) and loss of its nuclear localization are associated with the neoplastic progression of colon carcinoma. Proc Natl Acad Sci USA 90:3466-3470 (1993)

20) Endo K, Kohnoe S, Tsujita E, Watanabe A, Nakashima H, Baba H and Maehara Y: Galectin-3 expression is a potent prognostic marker in colorectal cancer. Anticancer Res $25: 3117-3121$ (2005)

21) Lauren $\mathrm{P}$ : The two histologic main types of gastric carcinoma: diffuse and so-called intestinal-type carcinoma. An attempt at a histo-clinical classification. Acta Pathol Microbiol Scand 64:31-49 (1965)

22) Hamilton SR and Aaltone LA: Pathology and Genetics of Tumors of Digestive System. Lyon, IARC Press, 2000. (World Health Organization Classification of Tumours)

[Received December 10, 2008: Accepted January 7, 2009] 